\title{
SCENARIOS FOR AN AUTONOMIC MICRO SMART GRID
}

\author{
Sylvain Frey ${ }^{1,2}$, François Huguet ${ }^{1}$, Cédric Mivielle ${ }^{1}$, David Menga ${ }^{2}$, Ada Diaconescu ${ }^{1}$ \\ and Isabelle Demeure ${ }^{1}$ \\ ${ }^{1}$ Infres \& SES Departments, CNRS LTCI-Télécom ParisTech, Paris, France \\ ${ }^{2} I C A M E$ Department, EDF R\&D, Clamart, France \\ ${ }^{1}\left\{\right.$ first_name.last_name\}@telecom-paristech.fr, ${ }^{2}\{$ first_name.last_name\}@edf.fr
}

\begin{abstract}
Keywords: Computing, Autonomic, Scenario, Vision, Decentralised Systems, Micro Smart Grids.
Abstract: Autonomic computing is a bio-inspired vision elaborated to manage the increasing complexity of contemporary heterogeneous, large scale, dynamic computer systems. This paper presents a series of scenarios relative to micro smart grids - district-size "smart" electricity networks. These scenarios involve situations where autonomic management approaches could provide promising solutions. They therefore appear as short stories of a possible autonomic micro smart grid, that illustrate the concepts of autonomic computing as well as the potential behind this vision. At the same time, these scenarios reveal open issues as well as novel perspectives on the future of micro smart grids.
\end{abstract}

\section{Motivation}

Autonomic computing is a vision that proposes conceptual and practical solutions to complexity management in computer systems (Horn, 2001). An autonomic computer system is defined by its ability to manage some of its own properties without the help of a human administrator. Namely, an autonomic system is capable of self-configuring, self-protecting, self-healing and self-optimising (Kephart \& Chess, 2003), relieving users from difficult management tasks.

So far, realisations of autonomic computing remain restricted to the management of simple, isolated systems: the domain still needs major contributions in order to create large-scale, heterogeneous, dynamic, open autonomic systems (Dobson et al., 2010). In particular, "bottom up" engineering methods propose to build such complex systems out of integration of simpler ones (Ulieru \& Doursat, 2011). However, unpredictable and possibly ambiguous interactions between autonomic subsystems make it difficult to comprehend the functioning of autonomic systems-of-systems.
The goal of this paper is to elaborate realistic scenarios for illustrating autonomic approaches, understanding the issues behind autonomic management and paving the way to an engineering process for complex autonomic systems (Frey et al., 2012). The domain of application chosen here is that of micro smart grids. After a short presentation of management requirements for micro smart grids, a series of scenarios will be exposed, that show the capabilities of autonomic systems and allow the analysis of required features for such possible autonomic solutions.

\section{Management requirements for Micro Smart Grids}

The constant increase of energy consumption makes electricity grid management expensive and complex. Differences between consumption troughs and peaks (e.g. during winter nights) lead national providers to over-dimension means of production in order to face ever-increasing but still rare maximal demand.

Meanwhile, production means and energy storages (solar panels, windmills, batteries, etc.) start being 
available for private use. Private users were so far exclusively consumers, now they may get the capability to participate to active load management. This situation raises the issue of integrating millions of production means and storage capacities under private control and therefore less controllable than traditional means. Indeed, for reasons of privacy and private property, direct interventions on equipments may often be impossible.

In order to cope with the challenges future electricity grids face, the smart grid vision proposes to couple the traditional electricity network with an information network allowing a "smart" management of the electrical load (Schiller \& Fassmann, 2010).

In a micro smart grid, each home participates to the network by selling its production or adapting its consumption according to the load. The system that manages the district grid gives live information on the load that determines current purchase and sale prices, according to customer contracts. Domestic productions are connected to consumptions, allowing a fine management of supply and demand, practically use by use. Thus, grid management is not only about limiting consumptions and maximising productions, but also scheduling these streams throughout the day, while taking local contexts and usages into consideration.

The vision of micro smart grids requires sophisticated management capabilities, in particular with respect to systems integration. Therefore this is a promising use case for autonomic computing to illustrate its capabilities while addressing concrete issues of the domain. The rest of this paper presents scenarios that detail how autonomic approaches could be a solution to micro smart grid management, with analysis of the technical requirements possible autonomic solutions should fulfil.

\section{3 "Heating System" Scenario}

Taking advantage of renovation work, Mark has invested in an autonomic underfloor heating. Thanks to a set of sensors and actuators, the system can monitor temperature in the room and receive instructions. A hot water tank allows accumulating and returning heat; this is done according to electricity rates, to Mark's orders, to the weather, to the actual temperatures in the rooms and to whether or not someone is present in the apartment. Mark has also activated a geo-location functionality: aware of Mark's position, the system can reduce temperature while he is out and ensures everything returns to normal when he comes back.
Mark may monitor his system live state: electrical consumption, desired temperatures compared to actual temperatures, money saved thanks to the water tank stock. The initial investment was substantial but Mark sees a difference on his bill and can be confident he will get return on investment. In addition to ecological considerations on energy consumption reduction, the regulator adapts to the occupants' desires via simple instructions. Reading his system history, Mark has realised that some occasional adjustments he did by hand - for instance, in his bathroom - have been learnt by the autonomic system that anticipates his morning shower since then.

Mark's inquiring mind has led him to play with his regulator's most advanced functionalities. He has been offered a choice of management profiles, favouring comfort, energy savings or bill reduction. After some experimentations, Mark eventually decided to follow an highly economical policy that reduced again his electricity bill. And when guests are expected, the system can automatically restore more suitable comfort conditions.

Analysis: This scenario raises the following issues:

- conflicts between objectives and an unpredictable context;

- necessity to anticipate the context, in particular, to develop learning features.

The equipment this scenario describes follows two contradictory objectives: "maintain temperature" versus "limit electrical consumption", in a dynamic, unpredictable context. This context must be monitored by the system via temperature sensors, measurements of the electricity consumption, grid load sensors, hot water stock probes, possibly presence sensors and geo-location system. Since measurements are imprecise by nature, the system must take possible errors (unidentified moves in a room, GPS failure, etc.) into account.

In addition to adapting to the context (temperature, domestic load) the autonomic system must be able to forecast it in order to achieve efficient management of its stock. In the scenario, the system must anticipate Mark's return home after work; it reloads its stock during the day, according to a consumption forecast for the night. This forecast may imply mid to long-term learning capabilities, in order to evaluate a standard night profile, possible variations of Mark's consumption, etc.

Unexpected events (unforeseen load peak, unusual order from the user, heat wave, cold wave, etc.) can challenge previous forecasts and threaten the 
system's functioning. However, it must be able to anticipate such variations and allow room for manoeuvre in order to face the unexpected with appropriate reactions. Sophisticated objectives can help the system solve the "margin of error versus optimisation" dilemma.

\section{4 "Room" Scenario}

Mark keeps testing new equipments in his flat and installed a sophisticated skylight featuring a controllable power-driven actuator and an autonomic controller. The latter fetches data produced by temperature and presence sensors in the room, reads objectives received by the heating system and follows local weather forecasts online. Aside from manual remote control, the controller proposes several programs for airing the room while maintaining its temperature.

After making sure that the skylight's functioning does not create security problems, Mark gives it order to open for 10 minutes a day, provided nobody is in the room and external conditions (temperature, rain, wind) do not threaten the room's temperature objective. In case unfavourable conditions persist, the skylight does not open and reports the event.

From studying its systems' journals, Mark has noticed that far from disrupting energy saving objectives, the skylights participates to temperature regulation: when outside temperature condition are favourable, the skylight opening eases the heating system's task.

Analysis: The scenario raises the following issues:

- integration of different equipments, on a small scale;

- collaboration between equipments in order to reach a common objective (here, temperature).

In order to simplify the scenario, we assume that the skylight's electrical consumption is negligible. In a simple case, integration between the heating system and the skylight may be minimal: the two equipments have no direct relation with each other, they simply interact through the physical world by influencing temperature in the room. This kind of integration, however limited, may lead to a valid system (Frey et al., 2010).

In a more advanced case, the equipments share predictions on their behaviour and take each other into account in their management logic. For instance, on a cold day, the skylight warns the heating system that a temperature drop may be necessary during the day, due to air renewal. The heating system answers with possible moments of the day it estimates it could face a temperature drop better (because its stock would be full, the grid's load should be low, Mark would be away). The two equipments eventually agree on the best solution and both reach their objectives with minimal disruption of each other ones'.

This "social ability" of autonomic equipments influences the way they are conceived from the very beginning, since they must be able to interact and cope with other systems that are potentially unpredictable and sources of errors. Thus, a properly designed autonomic systems should be able to run alone as well as to integrate a wider system, without having to undergo fundamental changes in its internal functioning. Therefore this "situatedness" of autonomic systems must be a key concern at the design phase.

\section{$5 \quad$ "District Grid" Scenario}

Mark's neighbour, Sal, just started her washing machine. Her flat not being equipped with means of production nor stocking, it is part of the consuming homes in the district.

Mark's flat is now equipped with an autonomic battery, in addition to existing equipments, therefore it is capable of stocking and reselling electricity. Detecting that the district grid load increases, the system performs an internal analysis, in order to decide what to do next. It gathers the following analysis: the battery is fully loaded, the flat's room has been aired already, no particular consumption is planned for the time being and a priori, the hot water tank should be able to face any thermal hazard by itself.

Thus, Mark's system decides to dedicate part of the battery's stock to external demand. The buyback rates are favourable to Mark; on her side, Sal benefits from cheaper local electricity.

Later on, Mark's unexpected return increases his home's consumption. Starting an intense housework session usually happening on week-ends, Mark has opened the windows wide. The heating system, unprepared, is compelled to spend its stock and even get power from the battery in order to restore a comfortable temperature. 
Mark's system not being able to provide the district grid without threatening its own interests (on both thermal and economical aspects) it ceases being a producer for its neighbours. Sal's home has lost a potential provider.

Analysis: The district grid is a typical example of distributed organisations bringing together concurrent entities with their own objectives. Each home tries to minimise its electricity bill and maximise its profits, although some less egoistic criteria may be involved as well. The district grid has no direct control on homes and their equipments; however it can encourage collaboration via advantageous fares. Buying and selling is decided at the home level, according to specific internal constraints, as shown in the scenarios.

The scenario introduces several issues:

- scaling up;

- global vs. local management;

- heterogeneity of the domains interacting.

The first of these difficulties excludes completely centralised solutions that would intend managing hundreds of homes and all their appliances with a single controller - which is, at best, an extremely difficult task. Furthermore, due to privacy respect, it is not conceivable that users entrust an external entity with managing their everyday appliances.

Global vs. local management is related to balancing interests of the different parties involved (homes, district and national grids). Variations of production means, average offer, average demand and energy costs are inevitable. Therefore the district grid has to feature a management system that equilibrates relations between the parties on the long run, via regulation means - energy fares and contracts, production means, storage. The policies driving this regulation depend on the rules (laws, institutions) that control the district grid.

Finally, heterogeneity of the domains interacting appears through the succession of perturbations at the end of the scenario: Mark unexpectedly returning home, disrupting the flat's temperature, hence local consumption increasing, hence district production decreasing, hence possibly Sal's system seeing energy prices rising. This chain of consequences illustrates unpredictable behaviours happening in heterogeneous distributed systems, and again pleads for decentralising decisions, in association with separation of concerns (usages management, temperature management, home energy management, district grid management).

\section{Conclusion}

This paper proposed a series of autonomic micro smart grid scenarios, illustrating the potential of autonomic systems and analysing requirements for possible autonomic solutions to micro smart grid management issues. Technical contributions, namely integration design patterns for autonomic management systems, have been published based on this work (Frey et al., 2012). Yet the authors hope that designing and exposing scenarios contributes to the understanding of micro smart grids and autonomic systems, unveiling conceptual and technical challenges that will be encountered by future engineers and researchers.

\section{REFERENCES}

Becker, B., Allerding, F., Reiner, U., Kahl, M., Richter, U., Pathmaperuma, D., Schmeck, H., Leibfried, T., 2010. Decentralized Energy-Management to Control Smart-Home Architectures. Lecture Notes in Computer Science, 2010, Volume 5974/2010.

Dobson, S.; Sterritt, R.; Nixon, P.; Hinchey, M.; 2010. Fulfilling the Vision of Autonomic Computing. Computer, vol.43, no.1, pp.35-41, Jan. 2010.

Frey, S., Diaconescu, A., Lalanda, P., 2010. A Decentralised Architecture for Multi-Objective Autonomic Management. In Proceedings of the 2010 Fourth IEEE International Conference on SelfAdaptive and Self-Organizing Systems (SASO '10).

Frey, S., Diaconescu, A., Demeure, I., 2012. Architectural Integration Patterns for Autonomic Management Systems. In Proceedings of the 9th IEEE International Conference and Workshops on the Engineering of Autonomic and Autonomous Systems (EASe '12).

Horn, P., 2001. Autonomic Computing: IBM's perspective on the State of Information Technology. IBM. http://www.research.ibm.com/autonomic/manifesto/

Kephart, J.O., Chess, D.M., 2003. The Vision of Autonomic Computing, Computer 36,1 (Jan. 2003).

Schiller, C.A., Fassmann, S., 2010. The Smart Micro Grid: IT Challenges for Energy Distribution Grid Operators. White paper, IBM.

Ulieru, M., Doursat, R., 2011. Emergent engineering: a radical paradigm shift. International Journal of Autonomous and Adaptive Communications Systems (IJAACS), 4(1): 39-60. 22 pages. 